\title{
Restoration of gait by functional electrical stimulation for spinal cord injured patients
}

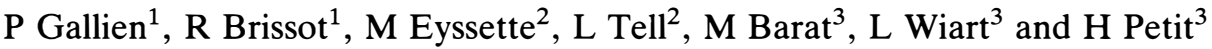 \\ ${ }^{1}$ Department of Rehabilitation, Hopital Pontchaillou, University of Rennes; ${ }^{2}$ Department of Rehabilitation, \\ Hopital Henri Gabrielle, University of Lyon, Saint Genis-Laval; ${ }^{3}$ Department of Rehabilitation, Hopital \\ Pellegrin, University of Bordeaux, France
}

\begin{abstract}
In this clinical study, we report the results of functional electrical stimulation for the ambulation of paraplegic patients without long leg braces (LLB), according to the Parastep approach. Of 13 SCI patients with complete neurological lesions included in this trial, 12 progressed to independent ambulation with the aid of the Parastep. The average walking distance was $76 \mathrm{~m}$, with a maximum of $350 \mathrm{~m}$, and the mean speed $0.2 \mathrm{~m} \mathrm{~s}^{-1}$. Compared to the situation with long leg braces, which in fact are given up by most paraplegic patients, long term home use seems to be much more important. Tolerance of this method is satisfactory. The psychological benefits of the device are remarkable. From this experience, it is concluded that this method is valuable for the restoration of standing and walking in the long term management of spinal cord injury patients.
\end{abstract}

Keywords: functional electrical stimulation; spinal cord injured; paraplegia; paraplegic locomotion; gait

\section{Introduction}

Since the report by Lieberson in $1961,{ }^{1}$ functional electrical stimulation has had an unquestionable development. Kantrowitz introduced it first, in the field of paraplegia. Then, various electrical stimulation techniques have been developed, starting from hybrid orthosis, ${ }^{2,3}$ with associated functional electrical stimulation (FES) to long leg braces (LLBs), to implanted devices. ${ }^{4-7}$ Following Krajl and Bajd's studies, ${ }^{8}$ Graupe and Kohn designed a transcutaneous FES device: the 'Parastep' ${ }^{9,10}$ Since the introduction of this device in France, in 1992, a multicentric evaluation has been conducted in three university rehabilitation departments (Rennes, Lyon and Bordeaux)..$^{11,12}$

We present here the results of this study on 13 neurologically complete SCI patients.

\section{Methods}

\section{Clinical and medical acceptance criteria}

Criteria for entrance to the protocol were: a stable orthoneurometabolic system; intact lower motor units; absence of ankylosis of the hip or knee; no cardiovascular or pulmonary disease; no epilepsy; no skin disease near the sites of stimulation; good standing tolerance; and adequate hand and finger control to manipulate the system. Pregnancy was also a contraindication.

\section{Subjects (Table 1)}

Thirteen paraplegic patients (11 males and two females), whose ages ranged from 17 to 42 years (mean:
$27 \pm 7$ years), with a clinically complete motor and sensory lesion, were studied. Details about the spinal cord injury appear in Table 1 . The clinical status of the patients did not present any contraindication for the use of the FES system.

The duration of the paraplegia ranged from 5 to 240 months, with an average of $60 \pm 20$ months. The aetiology was a motor vehicle accident in 12 patients, and a fall in one. The level of the lesion was thoracic in all of the cases, between T4 and T10.

\section{Device}

The system consists of a microcomputer controlled neuromuscular stimulator, powered by a rechargeable battery, providing approximately $2 \frac{1}{2} \mathrm{~h}$ of continuous use. The total weight is $500 \mathrm{~g}$. Six stimulation channels are available: two for the quadriceps, two for the peroneal nerve stimulation sites, and two for the gluteus or lower spinal muscles. Surface skin electrodes are connected by lead cables to the unit.

The device generates an alternating current monophasic symetrical wave form (with zero net charge), a pulse width of $300 \mu \mathrm{s}$, and a frequency of $24 \mathrm{~Hz}$. The amplitude of stimulation can be adjusted from 0 to $300 \mathrm{mAmp}$.

Standing is obtained by stimulating the quadriceps, coupled with the gluteus. During gait, the swing through phase is brought up by triggering the withdrawal reflex, while the controlateral lower limb is locked by stimulating the quadriceps.

A specially designed walker permits stability for ambulation. Built-in switches allow the commands and 
Table 1 Details of the subjects

\begin{tabular}{|c|c|c|c|c|c|c|}
\hline Subjects & Sex & Age & $\begin{array}{c}\text { Time from } \\
\text { SCI (months) }\end{array}$ & $\begin{array}{l}\text { Date of entry } \\
\text { into the study }\end{array}$ & Level & Aetiology \\
\hline 1 & M & 31 & 28 & May 92 & $\mathrm{~T} 7$ & Motor vehicle accident \\
\hline 2 & $\mathrm{~F}$ & 17 & 34 & June 92 & $\mathrm{~T} 4$ & Motor vehicle accident \\
\hline 3 & M & 21 & 5 & Sept 92 & $\mathrm{~T} 10$ & Motor vehicle accident \\
\hline 4 & $\mathrm{~F}$ & 36 & 240 & June 93 & $\mathrm{~T} 7$ & Fall \\
\hline 5 & M & 28 & 14 & August 93 & T5 & Motor vehicle accident \\
\hline 6 & M & 27 & 105 & Nov 93 & $\mathrm{~T} 4$ & Motor vehicle accident \\
\hline 7 & M & 31 & 33 & March 94 & T10 & Motor vehicle accident \\
\hline 8 & M & 25 & 18 & Sept 94 & T10 & Motor vehicle accident \\
\hline 9 & M & 30 & 21 & June 93 & $\mathrm{~T} 4$ & Motor vehicle accident \\
\hline 10 & M & 22 & 19 & July 93 & $\mathrm{~T} 8$ & Motor vehicle accident \\
\hline 11 & M & 42 & 204 & February 94 & T5 & Motor vehicle accident \\
\hline 12 & M & 27 & 54 & June 94 & T5 & Motor vehicle accident \\
\hline 13 & M & 23 & 14 & August 94 & $\mathrm{~T} 4$ & Motor vehicle accident \\
\hline
\end{tabular}

set levels of stimulation. A propylene ankle-foot (short orthosis) was used by patients to provide ankle stability during ambulation.

\section{Training}

The first sessions were focused on reinforcement of the quadriceps and the withdrawal reflex. Appropriate stimulation sites were determined for each subject to maximize the withdrawal response.

Regular assessment of quadriceps strength was noted in order to follow the muscular strength. Details about this assessment appear in the following paragraph. Standing was initiated when the strength of the quadriceps had reached $3.5 \mathrm{~kg}$. The quadriceps strength evaluation was performed according to the Sigmedics protocol. ${ }^{10}$ With electrostimulation, the quadriceps lifted up a definite weight, attached to the ankle, until there was complete knee extension, with the minimal level of intensity. Repetitive contraction was induced, increasing the weight of $500 \mathrm{~g}$ at each contraction. Afterwards, body balance, then successive phases of gait were performed between parallel bars. Ambulation out of parallel bars was introduced when progression using the bars was acquired and when the patient could initiate the steps by himself with the walker. This presumed that the patient had understood the functioning of the system.

\section{Evaluation}

Data come mostly from clinical recordings. Progression in standing and gait ability during the training, ambulatory performance, and functional use in daily life of the device were recorded. The quadriceps strength assessment was realised according to the protocol which has been previously described.

The repetition of the contractions also allowed us to test the endurance. FES quadriceps strength/endurance was estimated as the value of the maximal load lifted up by the quadriceps with the minimal electrical intensity. For safety reasons, applied loads did not exceed $10 \mathrm{~kg}$. Statistical analysis used for the quadri- ceps strength evaluation is the non-parametric Wilcoxon test, because of the small sample of population.

Thigh girth was also recorded.

Cardiovascular tolerance was assessed by measuring HR (beats $\min ^{-1}$ ), and BP, at rest and at the end of walking. In addition, reliability of the device, and side effects were supervised.

\section{Results}

\section{Gait training (Table 2)}

The standard number of 32 sessions, defined from the Sigmedics experience, was achieved in only three patients. The determinants for ending the training were acquisition of independence, satisfactory walking distance and, in a few patients, social reasons or medical complications. The total time and frequency of the training were personally adapted to each patient. Thus, most of the subjects ended their training before the 30th session.

The number of sessions was three to five per week. Each session lasted for about $2 \mathrm{~h}$. In most cases,

Table 2 Gait training

\begin{tabular}{lccc}
\hline Subjects & $\begin{array}{c}\text { First standing } \\
\text { (session) }\end{array}$ & $\begin{array}{c}\text { First walk in } \\
\text { parallel bars } \\
\text { (session) }\end{array}$ & $\begin{array}{c}\text { First walk with } \\
\text { Parastep } \\
\text { (session) }\end{array}$ \\
\hline 1 & 5 & 10 & 12 \\
2 & 1 & 2 & 2 \\
3 & 1 & 6 & 14 \\
4 & 1 & 4 & - \\
5 & 1 & 3 & 6 \\
6 & 1 & 3 & 8 \\
7 & 9 & 19 & 21 \\
8 & 1 & 3 & 5 \\
9 & 1 & 8 & 11 \\
10 & 1 & 5 & 7 \\
11 & 6 & 9 & 12 \\
12 & 6 & 6 & 10 \\
13 & 6 & & 17 \\
\hline
\end{tabular}


training was conducted on an outpatient basis; but for some patients living far from the centre, it was necessary to admit them as inpatients during the course of their training. Taking into account all of these factors, the majority of the patients could benefit from an accelerated training.

Three subjects had followed a previous programme with quadriceps stimulation, for 1 month, because of particular circumstances (distant home). The first standing with the Parastep was achieved quickly, most often within the first two sessions. The first steps with the walker were also quick, and took place around the tenth session, approximately 2 or 3 weeks after the onset of the programme. In one case, the patient did not complete the training, in spite of quick progress, for no understandable reason.

All but the above mentioned patient acquired independent ambulation with the Parastep.

One patient was unable to reach the stage of ambulation with the walker (case 4), but she was able to stand up by herself safely.

\section{Ambulatory performance (Table 3)}

Walking distances by the patients showed large differences, with a maximum of $350 \mathrm{~m}$ without rest.

The mean walking speed was about $0.2 \mathrm{~m} \mathrm{~s}^{-1}$, with a maximum of $0.6 \mathrm{~m} \mathrm{~s}^{-1}$, which is slow. Therefore, although the duration of independent walking was important for some of the patients, the proficiency of this gait was low, in regard to the distance covered.

The poorest performances were observed in the paraplegic patients with the longest history. Incidental diseases, such as urinary tract infections, induce a decrease of the fatigue threshold, and as a result a decrease of independent walking distance. Three patients had interrupted gait for several months (one for 5 months), without any decrease of their functional ability to walk, when they returned to training.

Quadriceps strength testing (Table 4)

A statistically significant increase of muscular strength was observed, showing noticeable variations among the patients, and also some difference between both quadriceps in a given individual. The thigh perimeter showed a mean increase of $5 \mathrm{~cm}$, and the cosmetic aspect of the entire lower limbs was much improved.

\section{Cardiovascular tolerance}

Cardiovascular tolerance was excellent. The mean heart rate increase, at the end of effort, was $41 \%$ of the rest frequency. The maximum observed frequency represented $68 \%$ of the cardiac theoretical maximum frequency. Blood pressure remained within the usual values in these patients; the maximum increase, at the end of the walk, was $40 \mathrm{~mm} \mathrm{Hg}$.

\section{Adverse effects}

Complications mostly affected the musculoskeletal system. Two patients complained of back pain perceived at the approximate level of the lesion. In these patients, obesity coexisted with a previous history of osteoarthritis of the spine. Two patients presented with a benign ankle sprain due to the fortuitous omission of

Table 3 Motor performance

\begin{tabular}{lcc}
\hline Subjects & $\begin{array}{c}\text { Maximal ambulation } \\
\text { distance }(m)\end{array}$ & $\begin{array}{c}\text { Speed } \\
\mathrm{ms}^{-1}\end{array}$ \\
\hline 1 & 188 & 0.3 \\
2 & 25 & 0.1 \\
3 & 24 & 0.05 \\
4 & 4 & $-\overline{3}$ \\
5 & 58 & 0.2 \\
6 & 100 & 0.1 \\
7 & 9 & 0.2 \\
8 & 350 & 0.2 \\
9 & 32 & 0.2 \\
10 & 80 & - \\
11 & 5 & $-\overline{2}$ \\
12 & 2 & 0.2 \\
13 & 40 & 0.2 \\
Mean & 73.25 & 0.1 \\
SD & 102 & \\
\hline
\end{tabular}

Table 4 Quadriceps strength testing (in $\mathrm{kg}$ )

\begin{tabular}{|c|c|c|c|c|c|}
\hline Subjects & $\begin{array}{l}\text { Right quadriceps } \\
\text { initial testing }\end{array}$ & $\begin{array}{l}\text { Left quadriceps } \\
\text { initial testing }\end{array}$ & $\begin{array}{l}\text { Right quadriceps } \\
\text { at discharge } \\
(P=0.0077)\end{array}$ & $\begin{array}{l}\text { Left quadriceps } \\
\text { at discharge } \\
(P=0.0077)\end{array}$ & $\begin{array}{l}\text { Time between } \\
\text { testings (days) }\end{array}$ \\
\hline 1 & 2 & 2 & 6.5 & 6.5 & 60 \\
\hline 2 & 2.5 & 2.5 & 7 & 10 & 120 \\
\hline 3 & 3.5 & 3 & 3.5 & 4.5 & 85 \\
\hline 5 & 7 & 7 & 10 & 10 & 30 \\
\hline 6 & 6 & 3.5 & 6.5 & 4 & 21 \\
\hline 7 & 0.5 & 1 & 1.5 & 5 & 90 \\
\hline 8 & 5.5 & 6.5 & 6.5 & 6.5 & 17 \\
\hline 9 & 5 & 5 & 10 & 10 & 196 \\
\hline 10 & 3.5 & 6 & 5 & 10 & 350 \\
\hline 11 & 3.5 & 8 & 5.5 & 8.5 & 35 \\
\hline Mean & 3.9 & 4.41 & 6.2 & 7.5 & 100 \\
\hline SD & 1.98 & 2.25 & 2.6 & 2.48 & 103 \\
\hline
\end{tabular}


their ankle-foot orthoses (AFOs). They recovered in a few days, without any effect on the furtherance of the training programme. One patient had a calcaneum fracture, certainly linked to the recovery of the upright position, and causing the arrest of the programme. This patient had been paraplegic for 17 years, and had given up standing.

A fracture of the sacrum was reported in another patient resulting from a fall, but without any functional consequence.

\section{Functional use}

Twelve patients, out of 13 , became independent for ambulation with the Parastep. One patient had the programme interrupted because of a fracture of the calcaneum. One patient gave up his training before the acquisition of a functional gait, although he was able to walk independently for $9 \mathrm{~m}$. Only eight, out of the 11 remaining patients, could get their personal device.

For the eight patients who possess their own device, we have at present a mean follow up of 15 months. Four are using their Parastep regularly, as an orthosis for walking at home. One patient has used the Parastep at home for $2 \frac{1}{2}$ years. One patient has given up after 1 year of daily use, and another uses it for static standing. The two remaining patients keep on being intermittent users in a rehabilitation centre.

In the cases where the Parastep is used regularly at home, we must note that it is not used to increase ambulation autonomy in daily life, but is used as an active means of exercise, in order to prevent complications of immobilisation, and to answer the desire to stand and walk.

In fact, in contrast to the lack of use for increasing locomotor autonomy, the psychological benefit of using the FES device seems remarkable. Although we have not documented it by specific tests, the psychological gain is obvious for the family and relations of the patient. Self esteem and self confidence appear to be greatly increased.

\section{Technical evaluation}

This device appears as reliable. No technical failure was noticed throughout this study. The training was quick and the learning simple. The lifetime of the disposable reusable electrodes was about 45 applications, from our experience. The lead cables required to be changed once a year, with regular use.

\section{Discussion}

It is well known that most paraplegic people give up their long leg braces, although it has been reported ${ }^{13}$ that $60 \%$ out of longstanding paraplegic people retain a strong desire to walk again. Functional electrostimulation can benefit only up to $20 \%$ of the paraplegic population. ${ }^{14}$

This group of patients, although small, provides interesting data. The device is reliable, easy to handle, and has a good cosmetic acceptance. Only one patient has given up during the training programme. Standing and gait are acquired in a few days. This observation confirms those of Graupe and Kohn. ${ }^{10}$

However, compared to normal gait, performance is modest. A great variability exists between patients: walking distance ranges from 4 to $350 \mathrm{~m}$. Marsolais ${ }^{5}$ has reported a maximum distance of $330 \mathrm{~m}$, after 3 years' use of his implantable system. Krajl ${ }^{8}$ and Graupe and Kohn ${ }^{10}$ mention performances close to ours, with a distance of $2 \mathrm{~km}$ for one patient. Hybrid orthoses ${ }^{2,3,15}$ offer a greater autonomy for walking, but with a cumbersome device, which is difficult to apply.

Speed remains the main limiting factor, whatever the technique used, because it is far from normal speed. ${ }^{4,8,10,16,17}$

Therefore the goal of FES synthesized gait restoration should not be an attainment of normal ambulation, but rather the realization of the benefits associated with biped locomotion as part of the management of spinal cord injury. The benefits of standing and muscular activity are considerable for these patients, along with subsequent reconditioning of cardiovascular function.

Muscular fatigue is an important limiting element, ${ }^{18}$ such as energy consumption, which has not yet been evaluated in our study. ${ }^{2,3,19,20}$ We have noticed an increase of muscle efficiency during training, with an improvement of the strength of the quadriceps and of motor performance. These data are consistent with those reported in previous studies. ${ }^{3,8,10,21}$

Few adverse effects are recorded in the literature. Hjeltnes and Lannem, ${ }^{16}$ like Graupe and Kohn ${ }^{10}$ reported one case of burn. Graupe noted one lower limb fracture due to a wrong use of the device, and several falls without any functional consequences. One of our patients, who was paraplegic for 17 years, sustained a calcaneum fracture; this demonstrates the osteoporotic risk secondary to paraplegia. Hangartner et $a l^{22}$ has shown recently the value of FES in preventing immobilisation osteoporosis. Another patient had a fracture of the sacrum after a fall but without any functional consequence.

Implanted systems are, nowadays, the providers of the greatest number of serious adverse effects, mainly infectious. ${ }^{5,6}$ Moreover, the lifetime of implanted electrodes is only about 20 weeks, which necessitates a new implantation.

Various clinical parameters must be carefully reviewed before admission into the programme: joint integrity; obesity, which can induce backache, by overloading the posterior joint of the spine; and osteoporosis in long standing paraplegic individuals. These parameters are not for us strict contraindications, but rigorous supervision is needed.

None of the patients use the device for daily life activities. This fact is observed, as a rule, for all ambulation techniques for paraplegic people. ${ }^{17}$ However this limited functional use, at the very beginning of this technique, seems widely counterbalanced by the tremendous psychological benefits, observed in most of our patients: better social rehabilitation, better per- 
sonal supervision of the complications of paraplegia, and thus, decrease of long term care costs.

The small dimensions and weight of the device, as well as the speed of putting it on, contrary to LLBs in hybrid techniques, can explain the small proportion of patients giving up the Parastep in the long term (five out of eight patients are home-users in our experience). One patient has been using the device for $2 \frac{1}{2}$ years. These preliminary data are consistent with those of Graupe and $\mathrm{Kohn}^{10}$ but must be confirmed by an extension of the study.

\section{Conclusion}

The Parastep is a reliable FES technology, which patients can easily learn. The results of this study show a quick progression in the acquisition of gait performance, and noticeable psychological benefits. The method is not indicated for all paraplegic people, and requires the subjects to be highly motivated.

The small size, along with the good tolerance of the device, are related to the long term use by patients (more than $50 \%$ in this study). Improvement of the technology ought to permit better efficiency, by improving gait speed, which at present is far from normal.

Further evaluation is needed to know precisely the energy expenditure generated by this method, and to define the selective aspects of the gait cycle, for which this expenditure can be lowered, with, as a result, an increase in the gait.

\section{Acknowledgements}

We are grateful to Anne Beaubras PT, Nicole Raux PT and Dominique Lainé PT who trained the patients with enthusiasm, patience and scientific rigour, and to the patients who entered the programme for their voluntary dedication. We would also like to thank Pierre Pain and the Sigmedics team: Paul Lavallée, Chairman, Franck Zeiss, Robert Habasevich, MSPT and Patrick Maher, for their trust in our rehabilitation team, their willing and continuous assistance, and their great help in this report. This study was supported by the INSERM-CNAM, the Fondation pour la Recherche Médicale, the University of Rennes (France), the University Hospital of Rennes, and the Ministry of Research and Technology (France).

\section{References}

1 Liberson WT. Functional electrotherapy: stimulation of the peroneal nerve synchronized with the swing phase of the gait in hemiplegic patients. Arch Phys Med Rehabil 1961; 42: 101-105.
2 Solomonow $\mathrm{M}$ et al. The RGO generation 2: muscle stimulation powered orthosis as a practical walking system for thoracic paraplegics. Orthopedics 1989; 12: 1309-1315.

3 Phillips CA. Functional Electrical Rehabilitation. Technological Restoration after Spinal Cord Injury. Springer: New York, 1991, p 203.

4 Holle $\mathrm{J}$ et al. Functional electrostimulation of paraplegics. Experimental investigations and first clinical experience with an implantable stimulation device. Orthopedics 1984; 7: $1145-1155$.

5 Marsolais EB, Kobetic R. Implantation techniques and experience with percutaneous intramuscular electrodes in the lower extremities. J Rehabil Res Dev 1986; 23: 1-8.

6 Marsolais EB, Kobetic R. Functional electrical stimulation for walking in paraplegia. J Bone Joint Surg Am 1987; 69: 728-733.

7 Rabischong P. Projet CALIES. Rencontre Autour du Blessé Médullaire. Masson: Paris, 1990, pp 265-271.

$8 \mathrm{Kralj}$ AR, Bajd T. Functional Electrical Stimulation: Standing and Walking after Spinal Cord Injury. CRC Press: Boca Raton, 1989, p 198.

9 Graupe D, Kohn KH. A critical review of EMG controlled electrical stimulation in paraplegic. CRC Crit Rev Biomed Eng 1988; 15: 187-210.

10 Graupe D, Kohn KH. Functional Electrical Stimulation for Ambulation by Paraplegics. Krieger: Florida, 1994, p 194.

11 Gallien $\mathrm{P}$ et al. Marche de synthèse chez le paraplégique par électrostimulation fonctionnelle: évaluations et résultats préliminaires. Actualités en Rééducation Fonctionnelle. Masson: Paris, 1993, pp 104-109.

12 Gallien $\mathrm{P}$ et al. Evaluation du pacemaker de marche selon la technique de graupe. La Marche Humaine et sa Pathologie. Masson: Paris, 1994, pp 215-220.

13 Petit $\mathrm{H}$ et al. Qualité de vie d'une cohorte de blessés médullaires 7 á 10 ans après l'accident. Evaluation de la Qualité de Vie. Masson: Paris, 1993, pp 175-188.

14 Jaeger RJ, Yarkony GM, Roth EJ, Lovell L. Estimating the user population of a simple electrical stimulation system for standing. Paraplegia 1990; 28: 505-511.

15 Isakof E, Douglas R, Berns P. Ambulation using the reciprocating gait orthosis and functional electrical stimulation. Paraplegia $1992 ; 30$ : 239-245.

16 Hjeltnes N, Lannem A. Functional neuromuscular stimulation in four patients with complete paraplegia. Paraplegia 1990; 28: 235-243.

17 Kantor C et al. Report on a conference on motor prostheses for workplace mobility of paraplegic patients in North America. Paraplegia 1993; 31: 439-456.

18 Rabishong E, Ohanna F. Modélisation de la fatigue musculaire induite par stimulation électrique de surface. Effet de l'apport vasculaire. Ann Réadapt Méd Phys 1993; 36: 205-217.

19 Leclaire $\mathrm{G}$ et al. Aspects bioenergétiques et adaptation cardiovasculaire chez le paraplégique appareillé par orthése de marche hybride. Résultats préliminaires. Actualités en Rééducation Fonctionnelle. Masson: Paris, 1992, pp 319-329.

20 Marsolais EB, Bennet GE. Energy cost walking and standing with functional neuromuscular stimulation and long leg braces. Arch Phys Med Rehabil 1988; 69: 243-248.

21 Sloan KE et al. Musculoskeletal effects of an electrical stimulation induced cycling programme in the spinal injured. Paraplegia 1994; 32: 407-415.

22 Hangartner TN, Rodgers MM, Glaser RM, Barre PS. Tibial bone density loss in spinal cord injured patients: effects of FES exercise. J Rehabil Res Dev 1994; 31: 50-61. 